\title{
REFLEXIONES EN TORNO AL EQUILIBRIO ECONÓMICO FINANCIERO
}

\author{
REFLECTIONS ON THE FINANCIAL ECONOMIC EQUILIBRIUM
}

\author{
Pierre Nalvarte Salvatierra \\ Rosselló Abogados, Perú \\ https:/ / orcid.org/0000-0003-4516-1716
}

\section{Resumen}

El equilibrio económico financiero es un concepto que, desde un punto de vista matemático financiero, puede ser determinado de manera objetiva, más allá de lo que prescriban las normas legales vigentes.

Es importante ser conscientes de ello. Aun cuando una norma o una cláusula contractual establezcan un mecanismo de restablecimiento del equilibrio económico financiero, puede que este no se alcance en la realidad, pues, para ello, en estricto, se tendría que mantener la rentabilidad esperada o el valor actual neto, de conformidad con el costo del capital aplicable.

El verdadero equilibrio económico financiero sí es tomado en cuenta cuando se evalúa una adenda a un contrato de APP o cuando se estructura una nueva APP. En caso de que se estipule en un futuro contrato un mecanismo que pretenda verdaderamente restablecer el equilibrio económico financiero, este se tendría que materializar elaborando un flujo de caja y observando la TIR o el VAN, incluso cuando esto resulte más complejo.

Palabras clave: equilibrio económico financiero, asociaciones público-privadas, tasa interna de retorno, valor actual neto, costo de capital. 


\begin{abstract}
Financial economic equilibrium is a concept that can be objectively determined, from a financial mathematical point of view, beyond what is prescribed by current legal regulations.

It is important to be aware of this. Even when a rule or a contractual clause establishes a mechanism for restoring the economic and financial equilibrium, it may not be achieved, because for this, strictly speaking, the expected profitability or the net present value would have to be maintained, in accordance with the applicable cost of capital.

The true economic and financial equilibrium is considered when evaluating an addendum to a PPP contract or when structuring a new PPP. If a mechanism is stipulated in a future contract that truly seeks to reestablish the economic and financial equilibrium, this would have to materialize by preparing a cash flow and observing the IRR or NPV, even if this is more complex.
\end{abstract}

Keywords: financial economic balance, public private partnerships, internal rate of return, net present value, cost of capital.

Pero llegando también el que había recibido un talento, dijo: Señor, te conocía que eres hombre duro, que siegas donde no sembraste y recoges donde no esparciste; por lo cual tuve miedo, y fui y escondí tu talento en la tierra; aquí tienes lo que es tuyo.

Respondiendo su señor, le dijo: Siervo malo y negligente, sabías que siego donde no sembré, y que recojo donde no esparcí. Por tanto, debías haber dado mi dinero a los banqueros, y al venir yo, hubiera recibido lo que es mío con los intereses.

(Mateo 25: 24-27)

\title{
1. Introducción
}

El presente artículo pretende realizar una aproximación objetiva, científica si es posible, al concepto del equilibrio económico financiero para, luego, analizar cómo ha sido regulada esta figura en el marco legal peruano y de qué manera ha sido recogida en los contratos de asociaciones público-privadas (APP). 
Una aproximación científica al concepto del equilibrio económico financiero implica poner énfasis en la diferencia entre una verdad objetiva — como una fórmula matemática - y el derecho — que se traduce en normas legales-. Esto puede explicarse con un ejemplo. De acuerdo con los parámetros médicos, la temperatura normal de una persona fluctúa entre 36 y 37.5 grados Celsius; a la temperatura superior a 37.5, y hasta 40 grados, se la denomina fiebre; y a aquella que supera los 40 grados se la llama fiebre alta o de urgencia. Ahora bien, en el supuesto de que exista una norma legal que establezca que la temperatura normal de una persona fluctúa entre 36 y 40 grados, es probable que el autor de dicha norma no tenga conocimientos básicos de medicina. Lo importante aquí es que no importa lo que diga la norma legal: la realidad no cambia.

Efectivamente, no importa que, desde el punto de vista puramente legalista, bajo una interpretación literal, una persona con 40 grados de temperatura se encuentre en perfecto estado. Lo real es que dicha persona tiene fiebre y requiere atención médica. Es importante no perder esto de vista y ser conscientes de la realidad, pues implica para los abogados ser humildes y reconocer que el derecho no cambia per se la realidad, sino que se construye sobre la base de esta. $Y$ a veces es posible equivocarse.

Partiendo de esta premisa, se procurará explicar, a continuación, cuál es el verdadero significado del equilibrio económico financiero, sin importar lo que digan las normas legales, sino únicamente desde el punto de vista de las finanzas, las cuales se construyen sobre las matemáticas.

\section{Lo que verdaderamente significa el equilibrio económico financiero}

Como su nombre lo indica, el aspecto esencial del equilibrio económico financiero lo determina el primer término: equilibrio. Es decir, debe existir una equivalencia entre lo que se invierte (egresos) y lo que se obtiene (ingresos).

Considerando que se está en el mundo de las finanzas, no se puede dejar de lado, de ninguna manera, otro concepto esencial: el costo de oportunidad del capital. Si alguien invierte S/ 100 hoy día y obtiene S/ 100 dentro de un año, no habrá, entonces, a causa del tiempo transcurrido, equivalencia entre dichas cantidades. El valor del dinero en el tiempo implica que S/ 100 de hoy no valdrán lo mismo dentro de un año o cinco. Lo racional es que todo inversionista exija un rendimiento, el costo del capital, por el transcurso del tiempo. 
Si, por ejemplo, el costo de oportunidad del capital fuera del $10 \%$, entonces, en caso de que una persona invierta S/ 100 el día de hoy, para que exista una equivalencia perfecta, ella debe obtener S/ 110 dentro de un año. Una cantidad menor de dinero como resultado de la inversión significa para el inversionista una pérdida desde el punto de vista de las finanzas. Lograr, en cambio, más de S/ 110 representa una ganancia por encima del costo de capital.

Ahora bien, para evaluar todo proyecto de inversión, se debe elaborar un flujo de caja. Esto no es más que una proyección de los egresos e ingresos que generará el proyecto. Luego será posible evaluar si este es rentable o no: es decir, si genera una rentabilidad por lo menos igual al costo de oportunidad del capital.

A continuación, un ejemplo sumamente sencillo de un flujo de caja. Supóngase que se invierten S/ 100 millones (periodo 0), y durante cinco periodos adicionales se obtienen ingresos de $S$ / 40 millones, mientras que los egresos ascienden a S 10 millones. El flujo de caja se muestra a continuación:

Flujo de caja (S/)

\begin{tabular}{|l|r|r|r|r|r|r|}
\hline Periodo & \multicolumn{1}{|c|}{0} & \multicolumn{1}{c|}{1} & \multicolumn{1}{c|}{2} & \multicolumn{1}{c|}{3} & \multicolumn{1}{c|}{4} & \multicolumn{1}{c|}{5} \\
\hline Inversión & -100.00 & & & & & \\
\hline Ingresos & & 40.00 & 40.00 & 40.00 & 40.00 & 40.00 \\
\hline Egresos & & -10.00 & -10.00 & -10.00 & -10.00 & -10.00 \\
\hline Flujo de caja neto & -100.00 & 30.00 & 30.00 & 30.00 & 30.00 & 30.00 \\
\hline
\end{tabular}

A simple vista se observa que la suma del flujo de caja neto obtenido del periodo 1 al 5 (S/ 30 millones cada año) es superior al monto de la inversión (S/ 150 versus S/ 100). Sin embargo, desde el punto de vista de las finanzas, no se pueden comparar directamente cantidades de dinero ubicadas en distintos momentos en el tiempo, pues ello implicaría desconocer el valor del dinero en el tiempo.

La bondad (o no) de este proyecto se puede medir, en un primer momento, a través de la tasa interna de retorno o TIR. En este ejemplo, la TIR del flujo de caja es de 15.24 \%; sin embargo, la TIR, por sí sola, puede ser insuficiente para determinar la viabilidad o conveniencia de un proyecto de inversión. Será necesario, entonces, tomar en cuenta el costo de capital aplicable al proyecto (esto dependerá, principalmente, del riesgo del sector de la actividad económica al cual pertenece). 
Asumiendo, para el mismo ejemplo, que el costo de capital es de $14.00 \%$, utilizándolo como tasa de descuento se puede obtener el VAN (valor actual neto) del proyecto. Este asciende a S 2.99 millones.

El VAN, también conocido como VPN (valor presente neto), resulta fundamental para tomar las decisiones de inversión:

Puesto que el objetivo de la administración financiera es aumentar el valor de las acciones, el análisis efectuado en esta sección lleva a la regla del valor presente neto: una inversión se debe aceptar si el valor presente neto es positivo y rechazar si es negativo ${ }^{1}$.

Estas dos medidas, la TIR y el VAN, sirven para medir objetivamente la viabilidad de un proyecto $\mathrm{y}$, por lo tanto, son el punto de partida para determinar su equilibrio económico. Existen otras medidas, pero estas son las más conocidas y utilizadas:

La evaluación del proyecto compara, mediante distintos instrumentos, si el flujo de caja proyectado permite al inversionista obtener la rentabilidad deseada, además de recuperar la inversión. Los métodos más comunes corresponden a los denominados valor actual neto, más conocido como VAN, tasa interna de retorno o TIR, el periodo de recuperación y la relación beneficio-costo².

\section{Cómo restablecer objetivamente el equilibrio económico financiero}

Continuando con el ejemplo anterior, si se supone que, por alguna razón o circunstancia, el Gobierno decide tomar la mitad de los ingresos del periodo 3, el flujo de caja sufriría una afectación que se puede medir en la TIR y el VAN, conforme se muestra en la página siguiente.

Si los ingresos del periodo 3 se reducen a la mitad y todo lo demás permanece igual, la TIR del proyecto habrá caído al 9.48 \%, mientras que el VAN será negativo (S/ -10.51 millones). Es decir, los flujos generados por el proyecto ya no serán suficientes para cubrir el costo del capital y, desde el punto de vista de las finanzas, el inversionista se encontrará ante una pérdida.

1 Stephen Ross, Randolph W. Westerfield y Bradford D. Jordan, Fundamentos de finanzas corporativas, 10. ${ }^{\mathrm{a}} \mathrm{ed}$. (Madrid: Mc Graw Hill, 2014), 221.

2 Nassir Sapag, Proyectos de inversión: formulación y evaluación (Santiago de Chile: Pearson Educación, 2007), 253. 
Flujo de caja (S/)

\begin{tabular}{|c|c|c|c|c|c|c|}
\hline Periodo & 0 & 1 & 2 & 3 & 4 & 5 \\
\hline Inversión & -100.00 & & & & & \\
\hline Ingresos & & 40.00 & 40.00 & 20.00 & 40.00 & 40.00 \\
\hline Egresos & & -10.00 & -10.00 & -10.00 & -10.00 & -10.00 \\
\hline Flujo de caja neto & -100.00 & 30.00 & 30.00 & 10.00 & 30.00 & 30.00 \\
\hline TIR & $9.48 \%$ & & & & & \\
\hline Costo de capital & $14.00 \%$ & & & & & \\
\hline VAN & -10.51 & & & & & \\
\hline
\end{tabular}

Cabe reiterar que se trata de un ejercicio puramente objetivo matemático, sin considerar lo que establezcan las normas legales o los contratos de APP.

Ahora bien, si se produjo ese perjuicio en el periodo 3, y en el periodo 5 se quiere compensar al hipotético inversionista, en este caso concesionario, ¿cuánto tendría que recibir este como compensación para que se restituya el equilibrio económico del proyecto?

Si alguien responde que se deben inyectar en el periodo 5 los S/ 20 millones que se sustrajeron en el periodo 3 , pues sería una respuesta equivocada desde el punto de vista de las finanzas, ya que este monto de ninguna manera restituiría el equilibrio económico financiero. Aun cuando se inyecten S/ 20 millones adicionales en el periodo 5, no se lograría que la TIR y el VAN sean iguales a los previstos originalmente en el proyecto:

Flujo de caja (S/)

\begin{tabular}{|c|c|c|c|c|c|c|}
\hline Periodo & 0 & 1 & 2 & 3 & 4 & 5 \\
\hline Inversión & -100.00 & & & & & \\
\hline Ingresos & & 40.00 & 40.00 & 20.00 & 40.00 & 60.00 \\
\hline Egresos & & -10.00 & -10.00 & -10.00 & -10.00 & -10.00 \\
\hline Flujo de caja neto & -100.00 & 30.00 & 30.00 & 10.00 & 30.00 & 50.00 \\
\hline TIR & $13.95 \%$ & & & & & \\
\hline Costo de capital & $14.00 \%$ & & & & & \\
\hline VAN & -0.12 & & & & & \\
\hline
\end{tabular}

Tal como se puede apreciar en el cuadro anterior, debido al efecto del valor del dinero en el tiempo, aun cuando se adicionen S/ 20 millones en el periodo 5, se obtendría una TIR del $13.95 \%$, inferior al $15.24 \%$ inicialmente 
previsto, así como un VAN negativo (S/ -0.12 millones), muy por debajo de los S/ 2.99 millones previstos originalmente.

Entonces, surge la interrogante, ¿cómo restituir verdaderamente el equilibrio económico financiero del proyecto? Pues bien, la respuesta desde un punto de vista matemático financiero es conceptualmente sencilla: se deberá inyectar una cantidad de dinero que haga que la TIR o el VAN vuelvan a sus valores originales.

Valor Presente Neto: desde una perspectiva teórica, el valor presente neto del proyecto al inicio de la concesión es la mejor medida de una posible alteración del equilibrio económico. El valor presente neto resume la expectativa generada al Concesionario al inicio de la Concesión, incluyendo los costos operativos, de mantenimiento, de inversión, distribuidos en el tiempo, así como el flujo de ingresos esperados ${ }^{3}$.

Por ejemplo, si lo que se quiere es que el VAN vuelva a ser igual al del proyecto original, el monto adicional que se tendría que inyectar en el periodo 5 ascendería a S / 25.98 millones. En efecto, si se adiciona esa cantidad a los S/ 40 millones de ingresos del periodo 5 , se tendrá como resultado que el VAN del proyecto volverá a ser de S/ 2.99 millones.

Flujo de caja (S/)

\begin{tabular}{|c|c|c|c|c|c|c|}
\hline Periodo & 0 & 1 & 2 & 3 & 4 & 5 \\
\hline Inversión & -100.00 & & & & & \\
\hline Ingresos & & 40.00 & 40.00 & 20.00 & 40.00 & 65.98 \\
\hline Egresos & & -10.00 & -10.00 & -10.00 & -10.00 & -10.00 \\
\hline Flujo de caja neto & -100.00 & 30.00 & 30.00 & 10.00 & 30.00 & 55.98 \\
\hline TIR & $15.13 \%$ & & & & & \\
\hline Costo de capital & $14.00 \%$ & & & & & \\
\hline VAN & 2.99 & & & & & \\
\hline
\end{tabular}

Para muchos autores, el VAN es el método más adecuado para medir la rentabilidad ( $\mathrm{y}$, por lo tanto, la viabilidad) de un proyecto:

3 Gonzalo Ruiz Díaz y Christy García, «Aspectos económicos e institucionales relativos al diseño de contratos de concesión viales», Themis Revista de Derecho 52 (2006): 251. 
Es el método más conocido, mejor y más generalmente aceptado por los evaluadores de proyectos. Mide la rentabilidad deseada después de recuperar toda la inversión. Para ello, calcula el valor actual de todos los flujos futuros de caja, proyectados a partir del primer periodo de operación, y le resta la inversión total expresada en el momento cero.

Si el resultado es mayor que cero, mostrará cuánto se gana con el proyecto, después de recuperar la inversión, por sobre la tasa i que se exigía de retorno al proyecto; si el resultado es igual a cero, indica que el proyecto reporta exactamente la tasa i que se quería obtener después de recuperar el capital invertido; y si el resultado es negativo, muestra el monto que falta para ganar la tasa que se deseaba obtener después de recuperada la inversión ${ }^{4}$.

Determinar el VAN de un proyecto es relativamente sencillo desde el punto de vista matemático. Esto es importante, porque permite obtener un parámetro objetivo que no está sujeto a discusión: si el VAN es positivo, el proyecto es viable; si el VAN era positivo y luego es negativo (o menor), el proyecto ha sido afectado:

Cualquier inversor o financiador prefiere tener su dinero hoy en vez de tener igual cantidad de dinero dentro de un año. Intuitivamente este es el concepto del VAN. (...)

El tratamiento matemático de cada flujo de caja es sencillo si utilizamos el software financiero incorporado en los ordenadores. El VAN es la diferencia entre el valor presente o valor actualizado de las entradas de caja -flujos positivos en un momento del tiempo- y el valor actualizado de las salidas de caja -flujos negativos-. Si es positivo el proyecto, es rentable y valdrá la pena estudiar su ejecución ${ }^{5}$.

De lo anterior es posible extraer dos lecciones:

a) Para restituir el equilibrio económico financiero no se puede pretender que la compensación de un perjuicio en un periodo dado (en el ejemplo, el periodo 3) sea igual a la misma suma, cuando esta es pagada en un periodo posterior (en el ejemplo, el periodo 5). Dicho en otras palabras, si lo que se dejó de ganar fueron S/ 20 millones, la compensación no puede ser por la misma cantidad, sino por un monto adicional.

4 Sapag, Proyectos de inversión, 253.

5 Diego Gómez y Juan Jurado, Financiación global de proyectos, project finance (Madrid: ESIC Editorial, 2001), 81 
b) Para restituir el equilibrio económico financiero es muy importante - mejor dicho, determinante-- el periodo en el cual se abona la compensación. Si esta se paga en el periodo 4, el monto por compensar sería diferente; en el periodo 6, la cantidad que se pague por concepto de compensación tendría que ser superior, de manera que se restablezca verdaderamente el equilibrio económico financiero.

\section{Lo que establece el marco jurídico peruano con respecto al equilibrio económico financiero}

Habiendo explicado en los numerales anteriores en qué consiste y cómo restituir objetivamente el equilibrio económico financiero de un proyecto de inversión, corresponde ahora saber cómo lo recoge la legislación peruana. Para ello, conviene centrarse en las normas concernientes a las APP, específicamente, en el Decreto Legislativo 1362 (en adelante, la ley) y su reglamento, aprobado mediante el Decreto Supremo 410-2018-EF, así como en sus directivas y lineamientos, cuando fuere el caso.

\subsection{Observancia en la formulación y estructuración de APP}

De acuerdo con la Ley de APP, el proceso que se sigue desde la concepción hasta la concreción de un proyecto vía APP se divide en cinco fases: planeamiento y programación, formulación, estructuración, transacción y ejecución contractual ${ }^{6}$.

La formulación abarca la etapa de diseño y evaluación del proyecto ${ }^{7}$. En esta fase se elabora, sobre la base de los estudios técnicos, el informe de evaluación ${ }^{8}$. Este contiene la información necesaria para definir si es técnica, económica y legalmente conveniente desarrollar el proyecto como APP9 ${ }^{9} \mathrm{La}$ evaluación económica financiera preliminar de este es parte del contenido obligatorio del informe de evaluación ${ }^{10}$.

6 Artículo 30.1 del Decreto Legislativo 1362.

7 Artículo 32.1 del Decreto Legislativo 1362.

8 Artículo 42.1 del Decreto Supremo 240-2018-EF.

9 Artículo 44.1 del Decreto Supremo 240-2018-EF.

10 Artículo 44.2, Numeral 4, Literal f del Decreto Supremo 240-2018-EF. 
Siendo claro que la fase de formulación comprende la elaboración del informe de evaluación y que dicho documento incluye, a su vez, una evaluación económica financiera, conviene ahora atender a los lineamientos aprobados que figuran en las guías y pautas para la evaluación económica financiera.

Cabe señalar que la DGPPIP ${ }^{11}$ del Ministerio de Economía y Finanzas (MEF) es competente para dictar este y otros lineamientos conforme a la ley ${ }^{12}$. Los Lineamientos para el desarrollo de las fases de formulación y estructuración en los proyectos de APP fueron aprobados mediante la Resolución Directoral 0052016-EF-68.01. El anexo A contiene específicamente los lineamientos para la elaboración del informe de evaluación.

Desde la sección $3^{13}$ de dicho anexo se puede apreciar la importancia del VAN para determinar la viabilidad de un proyecto.

En efecto, de acuerdo con la subsección 3.1, esta parte del análisis comienza por la elaboración de un flujo de caja. Luego, según se indica en la subsección 3.2, se debe evaluar si los ingresos del proyecto serán suficientes para garantizar su viabilidad; se considera, para ello, el costo de capital como tasa de descuento. Específicamente, los lineamientos recomiendan utilizar el costo promedio ponderado de capital (CPPC o WACC, siglas en inglés de weighted average cost of capital) como tasa de descuento para determinar la viabilidad del proyecto.

El CPPC o WACC se toma en consideración porque es la tasa que debe obtener una empresa para "compensar» el costo de la deuda y el costo de capital de sus accionistas:

Este CPPC tiene una interpretación muy sencilla: es el rendimiento total que debe ganar la empresa sobre sus activos actuales para mantener el valor de los títulos emitidos. También es el rendimiento requerido de cualquier inversión de la empresa que tenga en esencia los mismos riesgos que las operaciones actuales. Por tanto, si fuera a evaluarse flujos de efectivo de una expansión propuesta a las operaciones actuales, esta es la tasa de descuento que se usaría ${ }^{14}$.

El CPPC suele ser la tasa de descuento recomendada en la literatura financiera para evaluar la viabilidad de un proyecto:

11 Dirección General de Política de Promoción de la Inversión Privada.

12 Artículo 5.4 del Decreto Legislativo 1362.

13 Sección 3: Análisis de Brecha de Recursos y Asignación Preliminar de Riesgos.

14 Ross, Fundamentos de finanzas corporativas, 376. 
Elegir correctamente la tasa de descuento no suele resultar sencillo. Y de la tasa aplicada va a depender que el VAN del PF [Project Finance] analizado pueda ser más o menos elevado, o incluso negativo. La manera más extendida de calcular esta tasa se conoce como coste del capital o WACC (Weighted Average Cost of (apital) $)^{15}$.

En teoría, se puede utilizar otra tasa como tasa de descuento para determinar el VAN de un flujo de caja, como, por ejemplo, el costo de capital del accionista. Lo importante es que el costo de capital debe reflejar adecuadamente la rentabilidad exigida a una inversión realizada:

El costo de capital representa la tasa de retorno exigida a la inversión realizada en un proyecto, para compensar el costo de oportunidad de los recursos propios destinados a ella, la variabilidad del riesgo y el costo financiero de los recursos obtenidos en préstamos, si se recurriera a esta fuente de financiamiento. En otras palabras, la tasa de costo de capital debe ser igual a la rentabilidad esperada de un activo financiero de riesgo comparable, ya que los accionistas de la empresa estarían dispuestos a invertir en un proyecto si su rentabilidad esperada es más alta, en igualdad de condiciones de riesgo, que la que obtendría invirtiendo en activos financieros como, por ejemplo, acciones ${ }^{16}$.

Luego de esta primera evaluación, se pueden obtener uno de estos dos resultados, según la referida subsección $3.2^{17}$ :

- Un valor presente mayor o igual a cero significa que el proyecto no presenta una brecha de recursos.

- Un valor presente menor que cero significa que el proyecto presenta una brecha de recursos...

De esta manera queda demostrado que, desde la formulación de un proyecto de APP, se evalúa que este sea viable, considerando el indicador del valor presente (VAN o VPN) y que, para ello, se utiliza un costo de capital como tasa de descuento (específicamente el CPPC).

Obviamente, si el resultado de la evaluación anterior arrojase que el VAN es menor que cero, esto significaría que el proyecto no es sostenible por sí solo,

15 Gómez, Financiación global de proyectos, 82.

16 Sapag, Proyectos de inversión, 311.

17 Anexo A, Sección 3, Subsección 3.2, página 12, último párrafo. 
al existir una brecha de recursos que se podría cubrir mediante un cofinanciamiento $^{18}$.

En el apéndice $1^{19}$ del anexo A mencionado se desarrolla con mayor detalle la finalidad y los supuestos para estimar la tasa de descuento (CPPC); se precisa que esta sirve para descontar el flujo de caja del proyecto y estimar su valor presente; asimismo, que los supuestos para estimar el CPPC son:

- La estructura de capital

- La tasa del impuesto a la renta

- El costo de capital, que comprende el costo de la deuda y el costo del patrimonio.

$\mathrm{El}$ anexo B de los lineamientos bajo comentario contiene los Lineamientos para la elaboración del modelo económico financiero del proyecto en la fase de estructuración. Como resulta obvio, son aplicables en la siguiente fase de desarrollo de una APP: la estructuración. En esta fase continúan vigentes los conceptos señalados antes, es decir, que la viabilidad de un proyecto se determina en función del VAN.

En efecto, según la sección 2 del anexo B, la estructuración financiera del proyecto comprende la validación o actualización de la evaluación realizada en el informe de evaluación, pero tomando en consideración el análisis de riesgos asociados al proyecto, el análisis de los recursos disponibles para este (cuando se requiere cofinanciamiento) y la definición de los mecanismos de pago.

En su sección 3, el anexo B desarrolla en extenso las pautas para la elaboración de un «modelo financiero sombra». No es objeto del presente trabajo entrar en un análisis detallado de este, sino tan solo remarcar que lo sustancial del referido modelo - y de toda la estructuración en general- es determinar la viabilidad económica y financiera del proyecto, y para ello es indispensable elaborar los flujos de caja necesarios y encontrar su valor presente, descontándolos a una tasa apropiada.

En otras palabras, durante la formulación y estructuración de un proyecto de APP, se determina que este sea viable, lo cual constituirá el punto de partida para determinar el equilibrio económico financiero.

18 Artículo 31.1 del Decreto Supremo 240-2018-EF: «Cofinanciamiento es cualquier pago que utiliza fondos públicos, total o parcialmente, a cargo de la entidad pública titular del proyecto para cubrir las obligaciones establecidas en el respectivo Contrato».

19 Apéndice 1: Pautas para el análisis financiero durante la fase de formulación. 


\subsection{En la adjudicación de proyectos de APP}

En el artículo 58.4 del reglamento se establece que el adjudicatario de un proceso de promoción de una APP debe presentar obligatoriamente, antes de la firma del contrato, un modelo económico financiero del proyecto, de conformidad con los requisitos establecidos en las bases del respectivo proceso.

Cabe señalar que tanto el modelo económico financiero elaborado por el adjudicatario, así como el desarrollado por el OPIP ${ }^{20}$ correspondiente, pasarán a formar parte de la información que se incorporará en el Registro Nacional de Contratos de APP21.

En los Lineamientos para el desarrollo de las fases de formulación y estructuración en los proyectos de APP se indica, en la sección 3 del anexo B, que el modelo económico financiero por ser presentado por el adjudicatario debe contener, entre otros:

- Detalle de la estructura de capital.

- Tasa de retorno del capital invertido.

- Flujo de caja del proyecto.

\subsection{Incorporación en los contratos de APP de una cláusula de restablecimiento del equilibrio económico financiero}

En el artículo 37.1 del reglamento se dispone que en los contratos de APP se pueden incluir disposiciones sobre el equilibrio económico financiero:

Artículo 37. Restablecimiento del equilibrio económico financiero

37.1 Los Contratos pueden incluir disposiciones sobre el equilibrio económico financiero en las que se precise que su restablecimiento puede ser invocado por cualquiera de las partes, únicamente cuando éste se vea afectado significativamente debido al cambio de leyes aplicables, en la medida en que dichos cambios tengan impacto directo con aspectos económicos o financieros, vinculados a la variación de ingresos o costos asumidos por el Inversionista.

37.2 Una afectación se entiende como significativa cuando se hubiesen alcanzado los porcentajes que para tales efectos debe establecer el respectivo Contrato de APP. En estos supuestos, se restablece el equilibrio económico financiero al mo-

20 Organismo promotor de la inversión privada. Para proyectos de alcance nacional, generalmente el OPIP es ProInversión.

21 Artículo 139.1 del Decreto Supremo 240-2018-EF. 
mento anterior a la afectación significativa producida por los cambios en las leyes aplicables.

De acuerdo con el texto del citado reglamento, la incorporación de una cláusula de restablecimiento del equilibrio económico financiero está sujeta a las siguientes condiciones:

- El restablecimiento solo puede ser invocado cuando el equilibrio se vea afectado por un cambio de las leyes aplicables.

- El cambio en las leyes debe tener impacto directo en aspectos económicos o financieros vinculados a ingresos o costos.

- La afectación tiene que ser significativa.

- En el contrato se debe establecer el porcentaje para que una afectación se considere como significativa.

\subsection{Modificaciones a los contratos de APP}

En el artículo 55.1 de la ley se señala específicamente que, en el momento de modificar un contrato de APP, se debe mantener el equilibrio económico financiero:

Artículo 55. Modificaciones contractuales

55.1 El Estado, de común acuerdo con el inversionista, puede modificar el contrato de Asociación Público Privada, manteniendo su equilibrio económico financiero [cursivas añadidas] y las condiciones de competencia del proceso de promoción, conforme a las condiciones y requisitos que establece el Reglamento.

El mandato de la norma es claro. No se trata de una pauta o guía que el funcionario público puede elegir tomar en cuenta, sino de una instrucción. Se debe mantener el equilibrio económico financiero.

Lo anterior se ratifica en el artículo 134.1 del reglamento: cuando se modifica un contrato de APP, se debe mantener el equilibrio económico financiero:

Artículo 134. Modificaciones contractuales

134.1 Las partes pueden convenir en modificar el Contrato de APP, manteniendo el equilibrio económico financiero y las condiciones de competencia del Proceso de Promoción, procurando no alterar la asignación de riesgos y la naturaleza del proyecto. 
Quedando clara la mencionada obligación, surge un problema: ¿cómo verificar que con una adenda se está, efectivamente, conservando el equilibrio económico financiero de un contrato de APP?

Afortunadamente, ya existe al respecto una interesante experiencia adquirida. En aquellas adendas en las que se han producido variaciones importantes en variables como la inversión (CAPEX) y los costos de operación y mantenimiento (OPEX) —así por ejemplo, adendas que contemplan la incorporación de nuevas inversiones, que establecen la sustitución de inversiones, que acuerdan el adelanto o diferimiento de inversiones, entre otras-, el concedente ha efectuado un análisis del flujo de caja proyectado para verificar si, con las variaciones introducidas mediante la adenda, se mantenía a niveles similares la rentabilidad del inversionista. Es decir, si se mira desde el punto de vista del Estado, para verificar que el resultado de la adenda no sea producir una sobreganancia para el privado, sino mantener un nivel de rentabilidad similar.

A modo de ejemplo se cita aquí el caso de la adenda 2 al contrato de concesión de la red vial 4 (Pativilca-Santa-Trujillo-Salaverry-Empalme R01N).

La concesión de la red vial 4 comprende más de $350 \mathrm{~km}$ de vía. Entre sus principales aspectos incluye la ejecución de una segunda calzada, así como la construcción de las obras nuevas de los evitamientos Virú-Chao, Casma y Huarmey. La inversión referencial era de 286 millones de dólares ${ }^{22}$. El contrato se suscribió en febrero del 2009.

En el año 2016 se puso en evaluación la adenda 2, mediante la cual se iba a encargar al concesionario la construcción de la vía de evitamiento Chimbote. Para tener una idea de su importancia, basta con considerar que el costo total de la obra se había estimado en un monto que superaba los 174 millones de dólares ${ }^{23}$, es decir, más del $50 \%$ de la inversión proyectada originalmente.

El MTC $^{24}$ evaluó, entre otros aspectos, que se mantuviera el equilibrio económico financiero de la concesión, conforme lo ordenaba (y lo ordena) el marco legal vigente. En el informe 516-2016-MTC/25, la DGCT ${ }^{25}$ evaluó la necesidad y conveniencia de suscribir la adenda 2 . El anexo A de este documento recoge la evaluación que se hizo del modelo y del equilibrio económico financiero:

\footnotetext{
22 Ositrán, Evaluación económica de la concesión del tramo Pativilca-Puerto Salaverry (Red Vial 4, Autopista del Norte) (Lima: Gerencia de Regulación, 2010).

23 Numeral 3.2 de la Adenda 2 suscrita el 22 de julio de 2016.

24 Ministerio de Transportes y Comunicaciones, quien actuaba en calidad de Concedente.

25 Dirección General de Concesiones en Transporte del MTC.
} 
Los términos propuestos en la Cláusula 6.38 "Acuerdo de Ejecución de la Obra Vial Nueva No ofertada Vía de Evitamiento Chimbote" de la propuesta de la Adenda 2 del Contrato de Concesión son el resultado del análisis consensuado entre el Concesionario y el Concedente empleando un Modelo Económico, que se fundamente en la metodología del "flujo de caja descontado".

Se indica, más adelante, que el equilibrio económico financiero se mantiene sobre la base de la medición del VAN del proyecto:

El objetivo es compensar al Concesionario generando una Incidencia Económico Financiera (IEF) a su favor, para retribuirlo por la inversión que incurriría en la ejecución de las obras de Vía de Evitamiento sin afectar el Equilibrio Económico Financiero establecido en el Contrato de Concesión.

(...)

Los términos referidos mantienen el equilibrio económico financiero del Contrato de Concesión. Esto es en buena cuenta que el valor actual neto [VAN] del flujo de caja con Adenda 2 es igual o inferior al Contractual (sin modificaciones salvo las ya establecidas a través de la Adenda 1).

En el mismo anexo, se especifica también que se ha empleado la metodología del flujo de caja económico descontado al CPPC. Con esto se puede decir que queda absolutamente claro lo que se sostiene en el presente artículo: que para determinar si se mantiene (o restablece) el equilibrio económico financiero, se tiene que elaborar un flujo de caja y observar el VAN (o la TIR) del proyecto.

\section{Lo que establecen los contratos de APP}

Existen más de cien contratos de APP si se consideran los suscritos bajo el TUO de Concesiones (Decreto Supremo 059-96-PCM), la primera Ley de APP (Decreto Legislativo 1012), la segunda Ley de APP (Decreto Legislativo 1224) y bajo la ley actualmente en vigor, si solo se tienen en cuenta aquellos diseñados y estructurados por ProInversión (Agencia de Promoción de la Inversión Privada).

Obviamente, no es posible cubrir las cláusulas de equilibrio económico financiero incorporadas en todos esos contratos, puesto que ello excedería los alcances del presente trabajo. Sin embargo, se pueden tomar como muestra las 
cláusulas de equilibrio económico financiero incorporadas en el contrato de concesión del sistema de tratamiento de las aguas residuales de la cuenca del lago Titicaca, suscrito el 30 de octubre de 2019, bajo la vigencia de la actual ley.

En dicho contrato, el equilibrio económico financiero está regulado de las cláusulas 7.41 a 7.52 , inclusive. En la cláusula 7.42 se reitera, conforme a lo establecido en el reglamento, que las partes tendrán derecho al mecanismo de restablecimiento del equilibrio económico financiero exclusivamente por cambios en las leyes aplicables, excluyéndose cualquier otra causa para aplicar el mecanismo previsto en el contrato.

Para efectos de determinar cuándo es significativa la afectación, de conformidad con lo prescrito en el reglamento, en la cláusula 7.47 se señala que, si el porcentaje de desequilibrio supera el $10 \%$ (medido a través del resultado antes de impuestos), se procederá, entonces, a restablecer el equilibrio. Para efectuar esta medición, se debe comparar el estado de resultados del concesionario con un estado de resultados hipotético, en un escenario en el que no se haya producido un cambio en las leyes.

De acuerdo con la cláusula 7.48 del contrato bajo análisis, deberá ser el regulador el que determine el monto objeto de la compensación. Asimismo, la cláusula 7.52 establece que el concedente deberá efectuar las previsiones presupuestarias para que durante el primer trimestre del año siguiente se haga efectivo el pago correspondiente.

Finalmente, conforme a la cláusula 7.49 , se reconocerá la tasa Libor $+2 \%$ en caso de retraso luego del periodo máximo de abono pactado. Esto es, en caso de que recaiga en el concedente la obligación de compensar, luego de vencido el primer trimestre del año siguiente al cual se determinó el monto de compensación.

Una regulación bastante similar se encuentra en la mayoría de los contratos de APP suscritos durante los últimos diez años, específicamente los diseñados por ProInversión.

Ahora bien, en la regulación contractual antes resumida - la cual va en línea con lo establecido en el reglamento- se observa que el mecanismo de restablecimiento del equilibrio económico financiero no produce, desde un punto de vista estrictamente financiero, una verdadera restitución de dicho equilibrio. Esto se debe a las siguientes razones:

a) Para determinar que se ha roto el equilibrio económico financiero, se observa el estado de resultados, el cual no siempre es igual al flujo de caja de la empresa. 
b) El mecanismo de restablecimiento solo se activará cuando la afectación en el resultado antes de impuestos supere el $10 \%$. Es decir, si se produce una afectación del $9 \%$, no habrá derecho al restablecimiento del equilibrio económico financiero (no al menos en dicho periodo, pero es posible esperar que el efecto acumulado de dos o más periodos supere la barrera de 10 $\%$ ).

c) En el supuesto de que se supere la barrera del $10 \%$, para solicitar el restablecimiento del equilibrio económico financiero, se tendría que esperar al siguiente año de la producción del perjuicio (dado que se tiene que analizar al estado de resultados al cierre del año). Además, el concedente pagaría la compensación en el subsiguiente año, idealmente, dentro del primer trimestre. Es decir, aplicando estrictamente lo dispuesto en los contratos de APP, la compensación podría abonarse dos años después de producido el perjuicio, sin derecho al pago de intereses ni a una actualización del monto.

d) Finalmente, podría ocurrir que la compensación se pague inclusive después de vencido el primer trimestre del año siguiente. En ese supuesto, el concedente pagará solo un interés de Libor $+2 \%$, muy por debajo del costo de capital habitual de los concesionarios en una APP.

En consecuencia, la regulación contenida en los contratos de APP prevé un mecanismo de restablecimiento del equilibrio económico financiero (válido solo para el caso de cambios en las leyes aplicables) que no restituye, efectivamente, el equilibrio económico financiero desde un punto de vista objetivo, matemático-financiero.

¿Quiere esto decir, acaso, que no se debe respetar lo pactado en los contratos de APP en relación con el restablecimiento del equilibrio económico financiero? De ninguna manera. Por más imperfecto que sea el mecanismo contemplado en un contrato, lo pactado es ley para las partes y debe ser respetado. Así, por ejemplo, si en un contrato con prestaciones bilaterales se pacta que, ante el retraso en el pago de una parte, se le pagará a la otra una tasa de interés moratorio de $1 \%$ anual, dicho acuerdo es válido y tiene que respetarse, aun cuando en la práctica el pago de dicha tasa no compense el perjuicio sufrido por la parte fiel.

Pero lo importante, regresando a la regulación del equilibrio económico financiero en los contratos de APP, es reconocer que dichas cláusulas no cumplen estrictamente con restablecer el equilibrio económico financiero invocado. Y no lo hacen, pues no tienen como guía la restitución del VAN o de la TIR original del proyecto, a pesar de que, desde un punto de vista financiero, 
se sabe que alguno de esos indicadores debería ser tomado en consideración para restituir realmente dicho equilibrio.

Tan claro es que el VAN (que implica el concepto del costo de capital) o la TIR son los indicadores genuinos para comprobar que se mantiene o se restablece el equilibrio económico financiero que dichos indicadores son tomados en consideración en el momento de evaluar una adenda a un contrato de APP, como ya se ha explicado en una sección anterior.

\section{Lo que implicaría un verdadero restablecimiento del equilibrio económico financiero}

Si en un contrato se estipulase expresamente que una de las partes tiene frente a la otra la obligación de restablecer el equilibrio económico financiero por determinada causal o si se interpretase que ese deber fluye de un contrato cuando una de las partes perjudica a la otra con sus actos, la interrogante sería: ¿cómo se podría hacer efectivo el referido restablecimiento?

Pues bien, si este fuera el caso, conforme a lo expresado en este trabajo, se tendría que elaborar un flujo de caja y medirlo con el VAN o la TIR para verificar que, efectivamente, se restituya el equilibrio económico financiero.

Por supuesto que materializar un mecanismo como este resultaría más complejo que el procedimiento aritmético de comparar el estado de resultados con un estado de resultados hipotético. Es más complejo, sí, pero no imposible. De hecho, una evaluación similar se produce cuando se determina la viabilidad o no de una adenda a un contrato de APP.

En el caso de que se optara por medir que se ha alcanzado el restablecimiento del equilibrio económico financiero a través del VAN, habrá que determinar previamente cuál es la tasa de descuento o costo de capital que debería resultar aplicable. Existen para ello dos alternativas: que el costo de capital ya se encuentre fijado en el contrato o que el costo de capital deba ser determinado para cada caso en concreto.

Esto último, si bien requiere un trabajo adicional, es factible, y existen empresas especializadas que pueden realizar dicha estimación. Cabe señalar que usualmente se utiliza el CPPC como tasa de descuento.

Si se opta por medir el equilibrio económico financiero a través de la TIR, la tarea resultará algo más sencilla, puesto que ya no se requiere estimar el costo de capital, sino tan solo medir de manera directa la rentabilidad gene- 
rada por un flujo de caja. En esta situación, lo que se debe perseguir es que la TIR de un proyecto vuelva a ser la originalmente prevista.

\section{Conclusiones}

Existe una manera objetiva de determinar cuándo se mantiene (o restablece) el equilibrio económico financiero de un proyecto.

Lo anterior se realiza elaborando el flujo de caja y observando el VAN o la TIR del proyecto.

Si una norma o cláusula contractual establecen un mecanismo de restablecimiento del equilibrio económico financiero que no alcanza lo anterior (mismo VAN o igual TIR), no está cumpliendo verdaderamente su objetivo. El derecho no cambia la realidad.

En las APP, en el Perú, el equilibrio económico financiero es escrupulosamente observado en las fases de formulación y estructuración del proyecto de APP. Asimismo, es también estrictamente observado cuando se evalúa una adenda a un contrato de APP.

En el Perú, para analizar si se alcanza o conserva el equilibrio económico financiero en una APP, se recurre al flujo de caja descontado a una tasa de descuento adecuada, como por ejemplo, el CPPC.

En los contratos de APP, en el Perú, se incorpora una cláusula de restablecimiento del equilibrio económico financiero para un caso específico que, matemáticamente, no cumple con restablecerlo. El mecanismo es imperfecto.

Lo anterior no quiere decir, de ninguna manera, que no se cumplan las cláusulas de equilibrio económico financiero, pues el contrato es ley para las partes. Sin perjuicio de ello, es relevante tomar consciencia de lo imperfecto de dicho mecanismo. 


\section{Referencias}

Gómez, Diego y Juan Jurado. Financiación global de proyectos, project finance. Madrid: ESIC Editorial, 2001.

Ositrán. Evaluación económica de la concesión del Tramo Pativilca-Puerto Salaverry (Red Vial $n .^{\circ}$ 4, Autopista del Norte). Lima: Gerencia de Regulación, 2010.

Ross, Stephen A., Randolph W. Westerfield y Bradford D. Jordan. Fundamentos de finanzas corporativas. 10. ${ }^{a}$ ed. Madrid: Mc Graw Hill, 2014.

Ruiz Díaz, Gonzalo y Christy García Godos. «Aspectos económicos e institucionales relativos al diseño de contratos de concesión viales». Themis Revista de Derecho 52 (2006): 239-254.

Sapag, Nassir. Proyectos de inversión: formulación y evaluación. Santiago de Chile: Pearson Educación, 2007.

\section{Sobre el autor}

Máster en Derecho de la Contratación Pública por la Universidad Castilla La Mancha (España). Magíster en Administración (MBA) por la Universidad ESAN. Abogado por la Universidad de Lima. Socio de Rosselló Abogados (Rosselló Attorneys at Law). Profesor de la Maestría de Gestión Pública de la Universidad ESAN y de la Facultad de Derecho de la Universidad de Lima. 